\title{
Civilisations
}

Revue internationale d'anthropologie et de sciences

humaines

$51 \mid 2004$

Religions transnationales

\section{Un ethnologue chez les pentecôtistes du pays mossi}

A propos de : P.-J. LAURENT, Les pentecôtistes du Burkina Faso. Mariage, pouvoir et guérison, Paris, Karthala, 2003

\section{Sandra Fancello}

\section{OpenEdition \\ Journals}

Édition électronique

URL : http://journals.openedition.org/civilisations/697

DOI : $10.4000 /$ civilisations. 697

ISSN : 2032-0442

\section{Éditeur}

Institut de sociologie de l'Université Libre de Bruxelles

\section{Édition imprimée}

Date de publication : 1 janvier 2004

Pagination : 183-192

ISSN : 0009-8140

\section{Référence électronique}

Sandra Fancello, « Un ethnologue chez les pentecôtistes du pays mossi », Civilisations [En ligne],

51 | 2004, mis en ligne le 07 janvier 2009, consulté le 02 mai 2019. URL : http://

journals.openedition.org/civilisations/697; DOI : 10.4000/civilisations.697

Ce document a été généré automatiquement le 2 mai 2019.

(c) Tous droits réservés 


\section{Un ethnologue chez les pentecôtistes du pays mossi}

A propos de : P.-J. LAURENT, Les pentecôtistes du Burkina Faso. Mariage, pouvoir et guérison, Paris, Karthala, 2003

\section{Sandra Fancello}

\section{RÉFÉRENCE}

P.-J. LAURENT, Les pentecôtistes du Burkina Faso. Mariage, pouvoir et guérison, Paris, Karthala, 2003

\section{Des États-Unis à Ouagadougou}

1 C'est un ouvrage extrêmement complet que nous présente Pierre-Joseph Laurent, le spécialiste du pentecôtisme mossi, et qui vient compléter, outre de nombreux articles, un premier ouvrage (1998) centré sur une association paysanne (Wênd-Yam) qui faisait déjà une large place aux représentations et aux catégories de la pensée mossi, comme "l'invention de 'l'entente'» (wum taaba), et où apparaissait également l'une des problématiques fortes de l'auteur: le «groupe de la sortie du groupe ». Le présent ouvrage s'inscrit dans la continuité de l'approche monographique qui amena l'auteur à s'intéresser de plus près à ces «agropasteurs » ou pasteurs-paysans et à intégrer peu à peu le milieu pentecôtiste. Cette configuration singulière d'imbrication des mondes a permis à l'ethnologue d'opérer un glissement subtil, d'un objet à l'autre, sans rien perdre des éléments qui font du milieu pentecôtiste un « monde total ».

2 L'ouvrage s'ouvre naturellement sur une présentation historique de l'Eglise des Assemblées de Dieu, des Etats-Unis à Ouagadougou, qui souligne la place qu'occupa l'empire mossi dans les stratégies d'évangélisation des missionnaires américains, leurs stratégies d'implantation et le développement spectaculaire de cette Eglise dans un pays simultanément pénétré par l'islam. Les missionnaires, en effet, depuis la Sierra Leone où 
ils étaient arrivés tout d'abord, projetèrent de s'orienter d'emblée vers le pays mossi. La formation des premiers pasteurs mossi en fit bientôt, selon une expression de l'auteur, des «auxiliaires privilégiés », y compris hors de la zone de peuplement mossi. L'expansion transnationale des Assemblées de Dieu, soutenue par la contribution des pasteurs mossi, s'est faite d'abord en « se calquant sur les itinéraires de migrations mossi vers les pays côtiers ». Toujours encadrés par les missionnaires américains (et français), les pasteurs mossi n'en furent pas moins associés à l'implantation de la mission des Assemblées de Dieu dans des pays comme la Côte-d'Ivoire ${ }^{1}$, le Togo et le Bénin, et plus au nord, le Mali et le Niger. Le passage des missionnaires américains par la Sierra Leone avant d'atteindre le Burkina Faso illustre le rôle précurseur de diffusion et de «plaque tournante » des pays anglophones au sein de l'Afrique de l'Ouest. La zone d'expansion des Eglises pentecôtistes est aujourd'hui essentiellement concentrée sur un axe fort qui va de Lagos à Abidjan. Le Ghana et le Nigeria demeurent les principaux foyers de diffusion des Eglises nouvelles.

3 Quelques précisions cependant concernant l'implantation des Assemblées de Dieu au Ghana : cette Eglise est historiquement la première Eglise pentecôtiste entrée au Ghana ${ }^{2}$. La continuité avec le Burkina Faso est illustrée par le fait que ce sont deux des missionnaires parmi ceux installés au Burkina Faso, Margaret Peoples et Lloyd Shirer, qui partirent vers le Ghana au cours de l'année $1931^{3}$. D'abord basés à Kumbungu, près de Tamale, ils commencèrent à évangéliser le nord du pays (Yendi, Tamale, Walewale, Bawku, Bolgatanga), correspondant à la zone sahélienne des populations voltaïques (Dagomba et Mamprusi), s'inscrivant ainsi dans la continuité de l'évangélisation des Mossi du Burkina Faso telle qu'elle est décrite par l'auteur. Ils arrivèrent à Koumassi, en pays Ashanti, en 1944 et atteignirent la côte (Takoradi) en 1945, mais la croissance numérique des Assemblies of God fut très lente jusqu'à la fin des années 1970 et l'Eglise demeura cantonnée au nord et à la côte ouest du pays durant tout ce temps (Larbi, 2001 : 74). Elle fut intégrée au Ghana Evangelical Fellowship (créé en 1969, devenu le Ghana Pentecostal Council depuis 1981), la première institution rassemblant les grandes Eglises pentecôtistes de ce pays, comme la Church of Pentecost, l'Apostolic Church et la Christ Apostolic Church, mais ce n'est qu'au cours des années 1980 que l'Eglise connut un développement croissant. Elle serait aujourd'hui la troisième Eglise pentecôtiste du Ghana.

4 Au Burkina Faso, l'Eglise des Assemblées de Dieu n'est certes pas la seule Eglise, comme pourrait le laisser penser l'entrée monographique de l'auteur, mais elle est de loin la plus importante numériquement et "politiquement ", c'est-à-dire dans ses rapports avec la politique et ses modes d'implication dans la gestion des conflits. Elle est surtout la plus ancienne Eglise pentecôtiste de ce pays et en partie celle qui a engendré quelques autres dénominations comme la Mission Apostolique (1959), l'Eglise Apostolique (1965) et dans une moindre mesure, l'Eglise de Pentecôte (1969). En effet, ces trois dénominations furent créées successivement à partir du même groupe dissident des Assemblées de Dieu en 1959. Le " groupe des onze " se divisa une première fois en 1965 pour donner naissance à l'Eglise Apostolique et la Mission Apostolique adopta la dénomination «Eglise de Pentecôte " (1969) en conformité avec la Church of Pentecost du Ghana (anciennement Apostolic Church of Ghana ${ }^{4}$ ) à laquelle elle était affiliée depuis 1960. De ce point de vue, l'Eglise des Assemblées de Dieu, par son rôle précurseur, peut être considérée comme une sorte de matrice des Eglises qui apparurent plus tard dans le paysage religieux burkinabé. Plusieurs des fondateurs d'Eglises, comme le pasteur Bila Ouedraogo (Eglise Apostolique) 
ou Pinnoaga Yanogo (Eglise de Pentecôte) étaient d'anciens membres des Assemblées de Dieu.

5 Du point de vue historique, une approche comparative des modes d'expansion révèle quelques similitudes entre les Assemblées de Dieu du Burkina Faso et la Church of Pentecost du Ghana, qui est actuellement la première Eglise pentecôtiste du pays. En effet, alors que la première semble faire du Burkina Faso sa "plaque tournante» en essaimant ses pasteurs le long de la côte du Golfe de Guinée, la seconde, émergeant dans le sud, tente une percée vers le nord et progresse à la rencontre d'un islam en provenance du nord. Des pays comme le Burkina Faso, le Mali et le Niger forment à la fois une sorte de front musulman qui met au défi l'expansion des Eglises chrétiennes « du sud» et un point de rencontre où se cristallisent les enjeux d'une conversion "radicale " Assemblées de Dieu et l'Eglise de Pentecôte, le rapport de similitude est inversé : tandis que les premières peinent à s'imposer dans les pays côtiers (du moins à leurs débuts), la seconde ne réussit qu'une faible implantation dans le nord, du Ghana d'abord, et dans les pays sahéliens. Chacun profite donc d'une place d'exclusivité en son point de départ mais ne parvient pas à reproduire dans les pays voisins une implantation équivalente. La Church of Pentecost en particulier, basée à Accra, trouve plutôt ses relais auprès des capitales voisines, surtout Abidjan, tandis que les Assemblées de Dieu conservent, outre leur développement dans le Sud, des implantations fortes dans les pays du nord.

\section{Une Église « rurale » et « indigène »}

Contrairement à la nouvelle vague d'Eglises et de Mouvements pentecôtistes et néopentecôtistes qui privilégient le milieu urbain, l'Eglise des Assemblées de Dieu s'inscrit dans une tradition d'Eglises plus anciennes qui ont entrepris très tôt l'évangélisation des villages, soutenues en cela par le recrutement et la formation d'auxiliaires autochtones auxquels les missionnaires ont progressivement laissé la charge d'assemblées locales. Au Burkina Faso, la priorité donnée à l'évangélisation des Mossi a abouti à une forme «d'indigénisation » de l'Eglise qui en fait une Eglise «rurale » ou « indigène ». Ce point est commun à de nombreuses Eglises pentecôtistes d'origine missionnaire, c'est-à-dire héritées de l'évangélisation coloniale, et qui ont contribué à entretenir des relations d'exclusivité avec un groupe spécifique et à la fabrique d'identités chrétiennes ethnonationales comme dans le cas de la rencontre entre les Tswana et la London Missionary Society en Afrique du Sud, ou de la Church Missionary Society avec les Yoruba du Nigeria.

7 Replacée dans le paysage burkinabé, cette Eglise semble plus proche d'autres Eglises de la même mouvance comme l'Eglise de Pentecôte, d'origine ghanéenne, qui est essentiellement représentée par les Ashanti du Ghana. Mais en l'absence de migration ghanéenne au Burkina Faso, la prise en main de l'Eglise par les pasteurs mossi en fait un cas d'exception qui, à la fois s'affranchit de la tutelle ghanéenne et reproduit localement le modèle dominant de l'exclusivité ethno-nationale transposée en pays mossi. Sur ce point, les pratiques de l'Eglise de Pentecôte du Burkina Faso sont semblables à celles décrite par P.-J. Laurent concernant les Assemblées de Dieu et la priorité donnée à l'évangélisation des Mossi. Mais, si les pasteurs mossi, en tant qu'auxiliaires privilégiés, ont pu être à leur tour missionnaires des Assemblées de Dieu dans plusieurs pays, les pasteurs mossi de l'Eglise de Pentecôte ne contribuent pas à ce point à l'expansion missionnaire de leur Eglise, puisque ce sont essentiellement les pasteurs ghanéens qui se déplacent. Pour mieux cerner ce que peuvent être les parcours «initiatiques» et la 
formation des pasteurs, l'auteur y consacre une partie de son ouvrage, en essayant d'éclairer les ressorts de la " vocation de pasteur ».

\section{Portraits de pasteurs}

8 de Dieu au Burkina Faso, l'approche biographique, tout à fait novatrice, donne une dimension sociologique à ces portraits de pasteurs mossi. Ces récits biographiques évoquent, comme bien souvent, des épisodes de maladie ou de souffrance personnelle qui constituent l'événement "déclenchant » de la vocation, puisqu'ils sont interprétés, au terme, comme le signe de «l'appel divin». Choisis «en raison de leur puissance évocatrice et de leur valeur d'exemplarité ", ces récits peuvent en effet, comme le propose l'auteur, être appréhendés selon une succession de séquences régulières articulées autour d'éléments communs: aucun des portraits présentés n'évoque une conversion antérieure au christianisme. Tous étaient attachés à la religion traditionnelle, l'animisme, ou «combinaient l'islam et l'animisme» (pasteur Isaïe). Mais une maladie grave, interprétée comme une attaque en sorcellerie (pasteur Jonas/Adama) met à l'épreuve la puissance des fétiches, et bientôt, déçus par les recours magiques proposés par les devins et marabouts, tous s'accordent sur la puissance supérieure du Dieu chrétien. C'est donc dans la recherche de guérison que s'éprouve la puissance des fétiches ou leur inefficacité, comme en témoigne l'expérience du pasteur Jonas, du temps où il était Adama : «A présent que les kinkirse m'ont abandonné et que j'ai été attrapé par un sorcier qui est parvenu à cacher mon siiga, j'ai pensé que je devais rapidement me donner au Seigneur, sinon j'allais mourir ». Cependant, la pluralité des recours antérieurs à la conversion pentecôtiste montre que, si la conversion est perçue, a posteriori, comme un refuge, elle n'est pas le premier auquel on pense : les recours face à la maladie puisent successivement dans la religion traditionnelle et dans l'islam (ces deux champs du marché religieux étant souvent entremêlés). Ainsi, comme en témoignent les témoignages recueillis par l'auteur, la conversion pentecôtiste arrive souvent au terme d'une série de démarches, de consultations, voire de conversions multiples.

Comme pour beaucoup de jeunes Mossi, le passage par les plantations de la Côte-d'Ivoire s'inscrit dans leur parcours comme une étape: certains y font l'expérience de la conversion chrétienne (pasteurs Isaïe et Elisée). Pour chacun, il importe de montrer que la conversion vient au terme d'épreuves successives, de périodes d'errances ou d'un épisode de vie désordonnée (associée le plus souvent à la consommation d'alcool), de maladie ou de « folie », tel le pasteur Jonas qui se dit lui-même "presque fou » (p. 77). La sortie de crise est perçue comme l'intervention d'un pouvoir supérieur (qui « dépasse ») et salvateur. C'est «l'appel »: «j'ai compris que l'Esprit Saint voulait que je quitte le monde satanique (des esprits païens) », témoigne le pasteur Jonas. Mais la conversion, bien que perçue comme un "abri sûr", un refuge protecteur face aux puissances maléfiques, ne signifie pas la fin des épreuves pour le futur pasteur. Dans les premiers temps de sa mission, le jeune pasteur subit inévitablement une sorte de mise à l'épreuve dans l'évangélisation en milieu rural. Beaucoup subissent les attaques des villageois, qui sont autant de provocations, et se doivent d'y répondre par les manifestations de la puissance de l'Esprit Saint, en général des miracles. Ainsi témoigne le pasteur Isaïe, cité par l'auteur : «Depuis que je suis évangéliste, il n'y a pas un village où nous n'avons pas été attaqués. Les Burkinabé sont des gens de fétiches, des gens très durs : chacun veut

Civilisations, 51 | 2004 
tester sa puissance pour la mesurer à l'évangéliste et voir ainsi qui possède les meilleures protections » (p. 87).

10 Ces portraits de pasteurs ont la caractéristique, y compris pour l'enquêteur, d'être à la fois communs et exemplaires. Si la plupart des récits font état de ruptures dans le cours de la vie, voire d'épreuves personnelles (les récits sont eux-mêmes concentrés sur ces moments qui en donnent la structure générale), et insistent sur le caractère électif de l'appel, tous reposent sur les mêmes schémas: des récits de vie et de conversion séquentiels et articulés autour d'événements similaires. Que ce soient les pasteurspaysans mossi ou les leaders de mouvements transnationaux dont les biographies s'étalent sur les rayons de libraires évangéliques, tous intègrent à la base de leur récit des éléments similaires. En ce sens, l'approche de l'auteur n'est pas aussi réductrice qu'on pourrait le croire à la lecture de ces récits, elle montre au contraire, sans insister suffisamment peut-être, la construction a posteriori de ces types de récits. Ces portraits n'évoquent pas non plus la dimension transnationale des parcours de formation qui font aujourd'hui des dirigeants d'Eglises des "pasteurs-docteurs " ${ }^{6}$, voire des auteurs (de littérature évangélique). Dans la même lignée, le rapport de ces pasteurs à la littérature était à souligner car les Assemblées de Dieu disposent de presses qui, à partir de la Côted'Ivoire, jouent un rôle d'éditeur et de diffuseur de la littérature évangélique francophone équivalent à celui que jouent des éditeurs au Nigeria ou au Ghana pour les pays anglophones.

\section{Conversion et libération}

11 L'enquête ethnographique, qui repose sur une longue présence sur le terrain, nous livre une approche au plus près des modalités de l'appropriation des valeurs pentecôtistes par les Mossi, à travers la production de catégories nouvelles, hybrides, à la frontière des mondes mossi et chrétien. Si l'auteur ne s'attarde pas sur l'analyse de la traduction des notions du vocabulaire chrétien dans la langue vernaculaire et notamment les catégories du Mal (l'utilisation et la traduction des catégories de génies et d'esprits païens ou d'inspiration musulmane, comme les djinns, par les pasteurs mossi, en moore comme en français, mériteraient d'être davantage commentées), il concentre son analyse sur les catégories de la pensée et les représentations du changement individuel et social. La demande de guérison étant souvent au centre de la démarche de conversion, celle-ci s'exprime comme une libération de la souffrance et du Mal, libération temporaire, car la lutte, elle, est permanente. La conversion pentecôtiste revêt ici un double aspect : elle est à la fois une forme de distanciation du monde traditionnel et des contraintes liées aux règles coutumières, et une protection contre ses pouvoirs magiques (ou maléfiques). La diabolisation des pratiques coutumières ou traditionnelles amène le nouveau converti à considérer sa nouvelle religion comme le seul refuge face à la menace sorcière. La conversion pentecôtiste se présente donc à la fois comme un refuge d'où les fidèles tentent de se distancer des règles socio-économiques lignagères et comme le premier acte individuel qui vient s'opposer au «communautarisme villageois »: d'où le concept du "groupe de la sortie du groupe ", une formulation qui exprime subtilement comment la distanciation vis-à-vis du communautarisme villageois et de ses contraintes se traduit par l'intégration à une nouvelle communauté, celles des "frères et sœurs en Christ », tout autant réglementée et contraignante que la première, mais qui ouvre la voie à une forme 
de modernité sécurisée et sécurisante, face au contexte de « modernité insécurisée » dans lequel évoluent les jeunes mossi.

Dans ce contexte, les enjeux de la conversion tels qu'ils sont décrits par l'auteur ne rendent pas toujours compte de la violence des ruptures qu'elle implique, parfois pour le converti, avec sa famille et son entourage, tout un environnement social, ou comme dit l'auteur, un « réseau de dépendance ». La diabolisation de la société traditionnelle mossi se traduit par une perception globalisante et péjorative de certaines catégories de génies ou d'esprits (protecteurs pour les uns, diabolisés par les autres) qui sont aussi associées aux composantes de la personne mossi ${ }^{7}$. La « rupture totale avec le passé8» qu'implique la conversion et le rejet de tout un ensemble de représentations liées à la personne humaine, à l'identité mossi, profondément rattachées à l'origine du groupe et enracinées dans son histoire, constitue un retournement brutal et ferme pour l'individu à l'égard d'éléments inhérents à son identité culturelle et sociale. On comprend dès lors que la jeunesse pentecôtiste soit ainsi partagée, tiraillée, entre une forme d'aliénation et de dépendance à la société traditionnelle (et à ses pouvoirs magiques) et un désir de libération pour donner libre cours à l'expression d'ambitions plus individuelles. Le mariage en est une illustration et prend une signification particulière quand les jeunes gens refusant de se soumettre aux règles gérontocratiques qui régissent habituellement les alliances, se tournent vers les pasteurs et les possibilités qu'ils leur offrent de conclure un mariage dit par « consentement mutuel ». Cette prise de distance, et plus encore dans le cas précis du mariage, est à l'origine de nombreuses conversions chez les jeunes mossi. Ainsi, la majeure partie de l'ouvrage, qui est aussi la plus ethnographique, offre une analyse détaillée des modalités du mariage pentecôtiste tel qu'il est géré par les pasteurs mossi.

\section{Les ressorts du mariage pentecôtiste}

13 Pour comprendre l'importance du rôle joué par les pasteurs pentecôtistes dans les unions matrimoniales, il faut souligner que l'Eglise des Assemblées de Dieu est l'une des Eglises qui prône le mariage par « consentement mutuel ». Cette conception moderne du mariage suscite beaucoup d'intérêt chez les jeunes, notamment les jeunes femmes car, bien que le mariage par consentement mutuel soit la seule forme de mariage reconnue par le Code des personnes et de la famille entré en application en 1990, les autres formes de mariage héritées du droit coutumier, notamment le mariage par don et le lévirat, n'ont pas disparu et sont encore celles qui ont cours le plus souvent en milieu rural. La société mossi étant patrilignagère et gérontocratique, ce sont les chefs de lignage ou de famille qui régissent les unions matrimoniales. Ainsi, dans le cadre d'un mariage coutumier, une femme est supposée accepter un mari qu'elle n'a pas choisi. Le mariage ainsi conclu résulte davantage d'une alliance entre deux lignages que du choix des conjoints ${ }^{9}$. Une femme qui voudrait alors fuir un mari imposé trouve une issue dans la conversion pentecôtiste.

14 L'Eglise des Assemblées de Dieu est parmi les groupes pentecôtistes celui qui s'oppose le plus fermement au mariage coutumier, ainsi qu'au lévirat, une forme traditionnelle de redistribution des épouses du mari défunt parmi ses frères cadets ; (welghé da-bogho, litt. "partager les biens entre les héritiers", Alexandre, 1953: 70). La jeune femme ainsi " réfugiée » acquiert la double assurance d'être sous la protection d'un pasteur, insérée dans une nouvelle communauté de "frères et sœurs en Christ », et la possibilité d'y 
contracter une union matrimoniale selon son consentement. Bien entendu, ses formes d'union ne s'opposent pas aussi radicalement entre elles et la pluralité des appartenances religieuses autant que la pluralité des sphères de pouvoirs et des normes conduisent souvent les acteurs à la négociation et au compromis, bien plus qu'ils ne l'avouent. La présence des pasteurs pentecôtistes en milieu rural contribue à élargir le champ des possibles où progressivement, comme l'explique l'auteur, «cette sorte d'empilement de normes conduit à l'affaiblissement de toutes les sources d'autorité et de pouvoir par l'incapacité d'aucune à parvenir à imposer un monopole d'allégeance aux personnes », ou comme l'exprime un informateur, «les gens utilisent d'abord les lois qui rencontrent leurs intérêts!»(p. 94). C'est dans ce contexte producteur d'insécurité et face à une "utilisation opportuniste des règles qui régissent la vie en société », que les Assemblées de Dieu tentent d'imposer leurs propres règles afin « d'ajuster ensemble » (zems taaba) les modalités du mariage par consentement mutuel. Le pasteur lui-même organise les mariages de sa communauté, de ses «filles à marier ", reproduisant et régissant un véritable marché matrimonial : « avec l'âge et l'expérience, en véritable big man qu'il peut devenir ${ }^{10}$, le pasteur se trouve au centre d'un vaste réseau de relations de personnes vivant sous sa dépendance ou ayant sollicité des alliances auprès de filles de son Eglise " (p. 99). Cette réalité se traduit aussi chez l'auteur par une comparaison du pasteur au chef de lignage (buudukasma). Mais surtout, les règles du mariage protestant se heurtant parfois violemment aux enjeux des alliances traditionnelles, « finissent par accorder une place conséquente à certaines règles coutumières mossi", comme l'illustrent les descriptions de l'auteur. Car enfin, il faut dire que dans les types de mariage décrits par l'auteur, on consent surtout à ce qu'un jeune homme choisisse sa fiancée, laquelle, au terme d'une procédure engagée depuis longtemps auprès des parents de chacun, et avec l'accord de tous, ne peut que "consentir» à son tour. Si, au terme, il y a bien «consentement mutuel», il ne faut pas pour autant y voir l'expression d'un «libre choix" des conjoints, ou du moins de la jeune femme, qui contrasterait alors très fortement avec le mariage coutumier. L'apparente modernité du mariage protestant trouve ses limites dans le compromis avec une perception de l'individu (et de la femme) largement héritée de la société traditionnelle. La conception d'une « liberté » individuelle est rendue pensable «en prêchant inlassablement la désobéissance vis-à-vis des traditions, équivalentes (...) à des pratiques démoniaques" mais ne se traduit pas nécessairement par une liberté d'action des individus. Pour les jeunes mossi candidats au mariage protestant, pour qui «le désir d'émancipation du pouvoir des aînés et l'aspiration à une plus grande autonomie se focalisent d'abord autour du libre choix du conjoint », la conversion signe surtout le passage d'un groupe de dépendance à un autre.

\section{Guérison et délivrance}

15 L'impact des pentecôtistes mossi des Assemblées de Dieu en Afrique de l'Ouest est encore perceptible aujourd'hui à travers la figure de « croyants-guérisseurs » comme Elisée qui se déplace régulièrement en Côte-d'Ivoire. Mais le statut des croyants-guérisseurs n'est pas équivalent à celui des pasteurs. En l'absence de formation religieuse formelle et de statut hiérarchique au sein de l'Eglise, ils fondent leur réputation sur leur charisme personnel qui en fait, aux yeux des fidèles, des " prophètes » et les place en concurrence avec les pasteurs qui disposent, eux, d'un charisme dit « de fonction » : « le rôle ambigu tenu par le croyant-guérisseur au sein de l'institution pentecôtiste est mis en lumière par 
l'embarras devant lequel on se trouve quand il faut le désigner» (p. 313). Ils cumulent ainsi les dons de prophétie, de vision et de discernement des démons qui permettent aux croyants-guérisseurs de diagnostiquer les "maladies démoniaques». Mais surtout, en marge de l'orthodoxie de l'Eglise, dans un temple ou une cour, ils offrent aux fidèles une panoplie de recours et de pratiques comme les consultations individuelles ou la délivrance collective à propos desquels les postures «théologiques » de l'Eglise ne sont pas toujours claires. Ce point est commun à plusieurs autres Eglises pentecôtistes, donc certaines présentes au Burkina Faso, comme l'Eglise de Pentecôte, et rappelle singulièrement la controverse qui agite ces Eglises à propos des camps de prière et de délivrance. Pour Paul Gifford (1998: 108) la Church of Pentecost du Ghana est l'une des Eglises les plus étroitement associée à ce débat, compte tenu des nombreux camps qui exercent sous sa dénomination et des nombreuses dissidences qui sont associées à son histoire. Ces camps sont en effet très nombreux, et le débat est virulent, dans les pays côtiers comme le Ghana et la Côte-d'Ivoire où Elisée a passé une partie de sa vie et reçu une partie de sa formation "pratique " à travers sa collaboration avec les pasteurs ivoiriens et ghanéens. Les croyants-guérisseurs tels que les décrit P.-J. Laurent existent depuis longtemps dans ces pays à travers quelques figures de fidèles ayant reçu un don de l'Esprit et qui reçoivent en général chez eux pour prier à la demande. Mais peu d'entre eux parviennent à rendre leur activité autonome. Le plus souvent, ces croyantsguérisseurs exercent « sous couvert » de l'Eglise à laquelle ils sont rattachés ou associés, mais l'ambiguïté de leur statut reste entière. Cependant, la renommée d'un tel leader peut s'accroître au point que, dans la perception des fidèles, la dénomination de l'Eglise devienne secondaire et, à ce stade de développement, il n'est pas rare que le " prophèteguérisseur » en vienne à former le projet de se séparer de son Eglise-mère pour fonder sa propre Eglise. Ce cas de figure est à l'origine de plusieurs dissidences au Ghana et en Côted'Ivoire.

La signification des séances de délivrance collective ou individuelle est tout aussi ambiguë en ce qu'elles donnent un aspect clientéliste à la consultation des fidèles qui peuvent être de toutes confessions, y compris musulmans, sans que ces consultations et/ou guérisons ne se traduisent par une conversion immédiate. C'est un autre aspect des pratiques du croyant-guérisseur qui peut gêner l'Eglise en tant qu'institution qui se veut régie par des règles strictes et une « orthodoxie ». Dans la dernière partie de son ouvrage, l'auteur nous offre la description détaillée d'une consultation privée ainsi que d'une séance de délivrance collective. Le croyant-guérisseur Elisée semble être parvenu à se faire une place telle dans l'Eglise qu'il est le personnage principal d'une assemblée dans laquelle il exerce ses dons de manière hebdomadaire. L'auteur porte une attention particulière à la mise en scène $d u$ " plan de bataille » contre les démons et aux performances oratoires du " prophète »; en effet, le charisme du croyant-guérisseur est en partie fondé sur les récits qu'il fait des guérisons miraculeuses qui se sont opérées sous ses mains : «l'expérience singulière du croyant-guérisseur l'intronise dans un état de surnature dont il n'aura de cesse de témoigner à l'occasion de prières de délivrance (...) La narration biographique instaure son identité de guérisseur et prouve l'effectivité du don divin » (p. 319). Mais les descriptions de l'auteur, bien qu'extrê-mement précises, n'insistent pas suffisamment sur la violence du corps à corps fidèle-guérisseur dans ces séances de délivrance, ni sur la puissance gestuelle à laquelle sont soumis les corps «malades » dans la lutte contre les démons. Sans parler de l'épreuve psychologique que peut représenter l'aveu public de la contamination par le Sida, compte tenu de la stigmatisation dont sont généralement 
victimes les malades. Mais ces «techniques du corps» ne sont pas le propre de tel ou tel croyant-guérisseur et la violence de la délivrance est due aux représentations communes de la maladie comme possession démoniaque tout autant qu'à la métaphore de la «lutte».

Le thème de la délivrance, comme celui de la conversion et du mariage, permet à l'auteur de relever le défi d'une entrée localisée, monographique, au plus près des catégories de la pensée mossi, pour se centrer sur les voies de l'appropriation locale d'un mouvement par ailleurs transnational et transculturel. Ses analyses révèlent la tension entre les enjeux locaux de la conversion pentecôtiste et la dynamique transnationale d'un mouvement qui transcende ses lieux d'implantation à travers des figures de pasteurs-migrants ou de « croyants-guérisseurs » itinérants. L'Eglise des Assemblées de Dieu, comme bien d'autres Eglises pentecôtistes de sa génération, semble à la fois «indigène » et transnationale, comme peuvent l'être ses représentants, tel Elisée, formé dans les pays côtiers et prêchant dans la langue vernaculaire, le moore, dans un quartier périphérique de Ouagadougou. Enfin cet ouvrage constitue un outil majeur pour la connaissance des conditions d'ancrage du pentecôtisme dans ses rapports aux cultures locales, autant que dans ses modes de régulation des conflits villageois. L'analyse centrée sur le mariage "protestant» illustre bien, de ce point de vue, les types de compromis auxquels sont soumis les mouvements et les idéologies de la « modernité ».

\section{BIBLIOGRAPHIE}

ALEXANDRE, R. P., 1953, La langue möré, t. 1 \& 2, Dakar : IFAN.

BONNET, D., 1988, Corps biologique, corps social. Procréation et maladies de l'enfant en pays mossi (Burkina Faso), Paris : Editions de l'ORSTOM.

GIFFORD, P., 1998, African Christianity. Its Public Role, London : Hurst \& Cie.

LARBI, E. K., 2001, Pentecostalism. The Eddies of Ghanaian Christianity, CPCS (Centre of Pentecostal and Charismatic Studies), SAPC, serie 1, Accra-Ghana.

LAURENT, P.-J., 1998, Une association de développement en pays mossi. Le don comme ruse, Paris : Karthala.

MEYER, B., 1997, « 'Make a Complete Break with the Past'. Memory and Post-Colonial Modernity in Ghanaian Pentecostalist Discourse ", Journal of Religion in Africa, XXVI, 4, pp. 1-34.

POULET, E., 1970, Contribution à l'étude des composante de la personne humaine chez les moose. Etude principalement lexicologique, Ouagadougou : Ronéot.

TAPSOBA, I. F., 1990, Eglises et mouvements évangéliques au Burkina Faso 1921-1989, Ouagadougou.

YAMÉOGO, K., 1985, Essai sur l'implantation du Mouvement protestant au Burkina Faso. Evolution et impact des Assemblées de Dieu à Ouagadougou de 1921 à 1971, Ouagadougou : Université de Ouagadougou. 


\section{NOTES}

1. Les Mossi représentent l'une des communautés de migrants les plus importantes dans ce pays.

2. Devancée, entre autres, par les missionnaires catholiques (franciscains, 1482), les missionnaires de la Basel Society (1828) suivis des méthodistes (wesleyiens, 1835) et des missions anglicanes (1906).

3. En fait, on retrouve au Ghana un « couple Shirer » à la suite du mariage des deux missionnaires avant leur départ pour le Ghana. Ils y seront rejoints par d'autres missionnaires en 1932, 1934 et 1937 (Larbi, 2001, pp. 71-72 et note 56).

4. A la suite de sa dissidence avec l'Apostolic Church de Bradford en 1953, le missionnaire James McKeown en conserva la dénomination jusqu'en 1962. C'est la révélation de cette confusion qui précipita la séparation du groupe burkinabé (qui se croyait affilié à l'Eglise britannique, Yaméogo, 1985 : 37). Ceux qui restèrent affiliés à la Church of Pentecost de McKeown en adoptèrent le nom en 1969.

5. De l'islam au pentecôtisme, car il est fréquent dans ces pays que les nouveaux convertis soient en fait d'anciens musulmans.

6. Dans quelques centres de formation très réputés comme le Haggaï Institute ou d'autres implantés en Afrique anglophone, au Nigeria ou au Kenya par exemple.

7. Le siiga désigne une force spirituelle, le « double », sujet et objet de sorcellerie (Poulet, 1970), tandis que le terme kiima (de ki: mourir) désigne l'esprit d'un individu devenu ancêtre. Au pluriel, le terme kiimse désigne "l'autel des ancêtres" sur lequel on effectue les sacrifices destinées aux ancêtres, mais il peut désigner les ancêtres en général, «ceux que l'on ne nomme plus»(Bonnet, 1988: 35). Les kinkirsi, génies auxquels on attribue la fécondité, sont aussi considérés comme les compagnons (imaginaires) de l'enfant, ceux-là mêmes dont il est issu. Plus tard, ils pourront être évoqués pour illustrer la personnalité d'un individu (Bonnet, 1988).

8. L'expression est de Birgit Meyer (1997).

9. En milieu rural, la seule forme de mariage par « consentement mutuel » est l'enlèvement, avec la complicité de la jeune femme, ou la fuite. La situation se "régularise " généralement après coup auprès de la famille de la jeune femme. Le cas échéant, et plus rarement, en cas de refus de la famille, le couple s'enfuit.

10. La comparaison du pasteur avec un big man est un autre aspect des analyses de l'auteur à travers lequel il combine plusieurs modes d'approches, au croisement entre les logiques de la conversion pentecôtiste et les problématiques liées au développement rural.

\section{AUTEUR}

\section{SANDRA FANCELLO}

Sandra Fancello est allocataire de recherche à l'Ecole des Hautes Etudes en Sciences Sociales (EHESS) et prépare une thèse de doctorat sur Les dynamiques transnationales des pentecôtismes africains. Elle est associée à l'Unité de recherche "Constructions identitaires et mondialisation » de l'IRD (Institut de recherche pour le développement). 